\title{
Resistance to Infection: Prospects for Therapeutic Manipulation
}

\author{
RICHARD B. JOHNSTON, Jr.
}

\author{
Department of Pediatrics, University of Pennsylvania School of Medicine, and The Children's Hospital of \\ Philadelphia, Philadelphia, Pennsylvania 19104
}

I have been charged to discuss some aspect of contemporary science in the general area of infectious diseases and to bridge that contemporary science to the future. The contemporary element is easy. Projecting into the future is not. Scientists and scholars have been notoriously bad at extrapolating from their present state of knowledge. For example, consider the following comments from established experts, all made around the time of the founding of the American Pediatric Society 100 years ago.

Note this clear-cut judgment on vaccination by an authority of the day: "Cowpox does not exercise any specific protective power against human smallpox." (E. M. Crookshank, Professor of Comparative Pathology, Kings College, London, 1889) (1).

Consider this comment by the inventor of the phonograph: "The phonograph is not of any commercial value." (Thomas A. Edison, 1880) (1).

The New York Times, then as now an expert on everything, had this to say about the art of Degas: "Degas is repulsive." (The New York Times, April 10, 1886) (1).

And finally, consider how dated is this particular comment of 100 years ago: "When a woman becomes a scholar there is usually something wrong with her sexual organs." (Friedrich W. Nietzsche, 1888) (1). This one would not go over particularly well in my household or at Grand Rounds.

So I hope you will forgive me, then, if I am rather cautious as I try to predict the future and if I focus more on present knowledge that should be the basis for future development.

The subject of this general section of the symposium is infection. This particular presentation will consider the host's part in that phenomenon, that is, resistance to infection, or what the host does back to the infecting organism.

To date, the most effective control of infectious diseases has come through their prevention, especially through immunization. Certainly, further efforts must be made with vaccines to achieve broader protection and easier dissemination to developing countries. But I wish to suggest that, in the unfortunate but common circumstance in which prevention is not possible and antibiotics are ineffective, treatment can be achieved by manipulation of the immune system. I will try to trace for you in summary form the evolution of current knowledge of the therapeutic "up-regulation" of one component of that system, namely, phagocytic cells.

The professional phagocytes, neutrophils, monocytes, and tissue macrophages, constitute the best-defined cells that act in the effector limb of the immune response. Cytotoxic lymphocytes and natural killer cells are other constituents of this system but are less well understood. One reason for the better definition of the phagocytic cells is the existence of experiments of nature within these cells lines, for example, chronic granulomatous disease (CGD) (2) and the leukocyte adhesion defect resulting

Correspondence and reprint requests: The Children's Hospital of Philadelphia, 34th and Civic Center Boulevard, Philadelphia, PA 19104.

Supported in part by Grant AI 24782 from the National Institute of Allergy and Infectious Diseases. from deficiency of the CD11-CD18 family of membrane glycoproteins (3). Members of the American Pediatric Society and the Society for Pediatric Research have been leaders in elucidating the relationship between the molecular defects and the predisposition to infection in these disorders, and the combined annual national meeting of these societies has been perhaps the single most important forum for presenting that work.

My hypothesis and the theme of this presentation can be summarized as follows: Treatment of infectious diseases in the future, at least under certain circumstances, will involve a capacity to manipulate the function of phagocytic cells. Full achievement of this capacity will depend upon our understanding the biochemical basis of the functions that we hope to modify.

There is a caveat, however. Inflammation is a double-edged sword. The phagocytic functions necessary for survival against infection can also damage tissue. Because this is the case, however, understanding the biochemical basis of phagocyte function also offers hope for the control of inflammation.

I would like to develop this story by showing you examples, both examples of the manipulation of the function of phagocytic cells and examples of attempts to understand the biochemical basis for the changes induced. I will focus on one particular aspect of phagocyte function, the so-called respiratory burst. That phenomenon is summarized in simplified form in Figure 1.

In this process, a microorganism coated with antibody, and usually also complement, interacts with receptors on the plasma membrane of the phagocytic cell and stimulates an enzyme complex associated with the plasma membrane. This enzyme system takes oxygen from the surrounding medium into the plasma membrane. It inspires, if you will, and reduces the oxygen by addition of one electron to yield superoxide anion $\left(\mathrm{O}_{2}{ }^{-}\right)$. Two molecules of $\mathrm{O}_{2}^{-}$interact with each other, in a dismutation reaction, to give hydrogen peroxide. Peroxide and $\mathrm{O}_{2}{ }^{-}$interact in the presence of heavy metal, especially iron, to yield a particularly potent oxidant, said to be the most potent oxidant recognized yet in nature, generally schematized as $\cdot \mathrm{OH}$ and termed hydroxyl radical (4).

The respiratory burst enzyme complex is presently under intense study. The complex is conceptualized to consist of two to four components that assemble as an active enzyme in the plasma membrane after the cell has been stimulated (4). The best defined constituent is a unique cytochrome, so-called cytochrome $b$, which is felt to make the final transfer of the electron onto oxygen to give $\mathrm{O}_{2}^{-}(5)$. Phagocyte cytochrome $b$ is missing in the classic $x$-linked form of CGD. Data also support NADPH as the source of electrons and the presence of a flavin-dependent enzyme in the system.

The work of Stuart Orkin, who is a member of the APS, with Mary Dinauer and associates, has located the gene for the major piece of cytochrome $b$ (there are two chains) on the short arm of the $x$ chromosome (Xp21.1) (6). This protein exists primarily in the membrane of specific granules in resting human neutrophils. When the cell is stimulated, the cytochrome is transferred to the 
PLASMA MEMBRANE MICROORGANISM

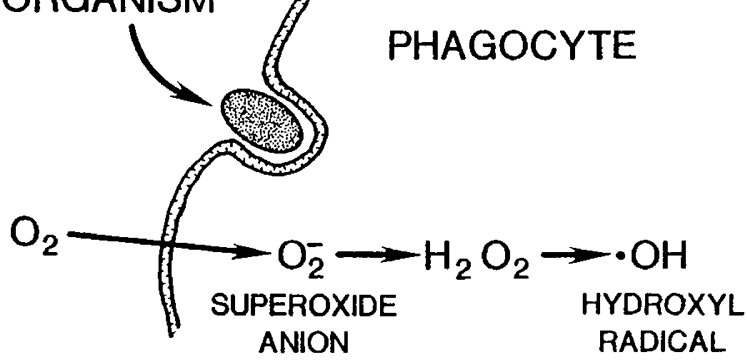

Fig. 1. The phagocytosis-associated respiratory burst.

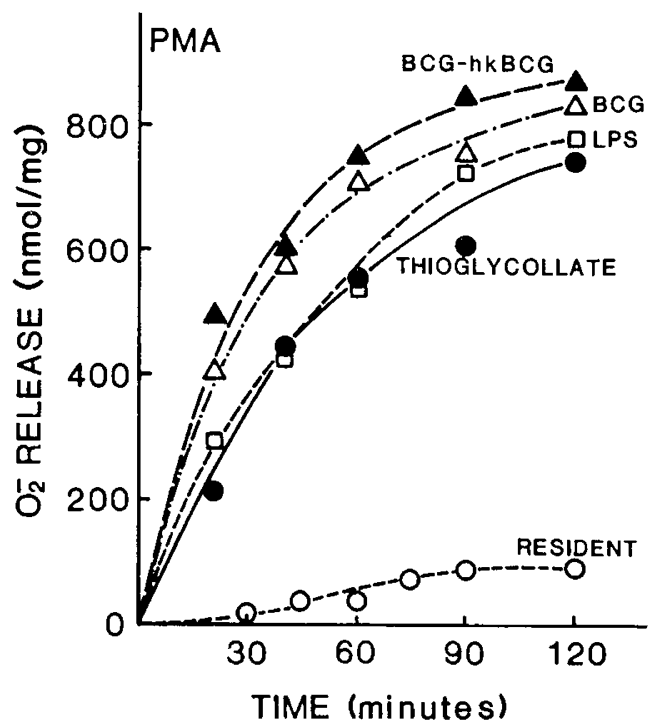

Fig. 2. Extent of $\mathrm{O}_{2}^{-}$release by mouse peritoneal macrophages plotted as a function of time, with PMA as stimulus. Cells were elicited by intraperitoneal injection of LPS or thioglycollate, or obtained from animals infected with the BCG strain of myobacterium, without or with the further injection of heat-killed (hk) BCG. (Modified from Ref. 8, with permission)

plasma membrane as the specific granules discharge their contents (5). There are one, two, or maybe even three additional factors present in the cytosol of the resting cell. One of these could be the flavin-dependent enzyme identified in cell membranes from stimulated cells, but this relationship is unclear. In this system, then, an electron is removed from NADPH and transferred to oxygen. The enzyme system is generally referred to as the NADPH oxidase or the respiratory burst oxidase.

Let us move next in the story to activated macrophages. By definition, a macrophage is activated if it has an increased capacity to kill microorganisms or tumor cells (7). About 10 years ago, the following question arose: Could the increased killing capacity of activated macrophages be ascribed at least in part to an increase in the respiratory burst? The experiment shown in Figure 2 suggested that it might.

The figure indicates that macrophages obtained from mice infected with the bacille Calmette-Guerin (BCG) strain of mycobacteria or injected with an inflammatory agent [endotoxin (lipopolysaccharide, LPS) or thioglycolate] have a markedly greater capacity to release $\mathrm{O}_{2}{ }^{-}$than do resident (normal) macrophages when the cells are stimulated. In this experiment, the stimulus was the phorbol diester, phorbol myristate acetate (PMA). Unstimulated macrophages of any sort do not release $\mathrm{O}_{2}^{-}$.
For the sake of clarity, this up-regulation has been defined precisely by the term "priming." Webster's fourth definition of the transitive verb "to prime" is, "to put into working order by filling or charging with something," as for example, in charging a pump. As applied to phagocytes, "primed" refers to the state or condition of a phagocyte in which the cell is modified so that it responds to phagocytosis or membrane perturbation, particularly from PMA, with a marked increase in the respiratory burst. This phenomenon is demonstrable only upon stimulation. It is a manifestation of macrophage activation but is not the same thing as macrophage activation, which includes many other biochemical and functional changes (7).

It seemed important to reproduce priming with a defined priming molecule in vitro to be able to study the phenomenon under controlled conditions. In the system shown in Figure 3, normal resident macrophages were preincubated overnight with LPS or muramyl dipeptide (MDP), a constituent of the cell wall of most bacteria, in the concentrations shown. Exposure to $\mathrm{ng} / \mathrm{mL}$ concentrations of either LPS or MDP primed the macrophages for a much greater respiratory burst, measured as release of $\mathrm{O}_{2}^{-}$.

Priming of macrophages has been achieved with other agents, which are summarized in Table 1. Some of these agents have therapeutic potential, particularly interferon- $\gamma$, and perhaps also colony-stimulating factor-granulocyte, monocyte (CSF-GM) (7, 10-13). Those in the second category may be active at local sites

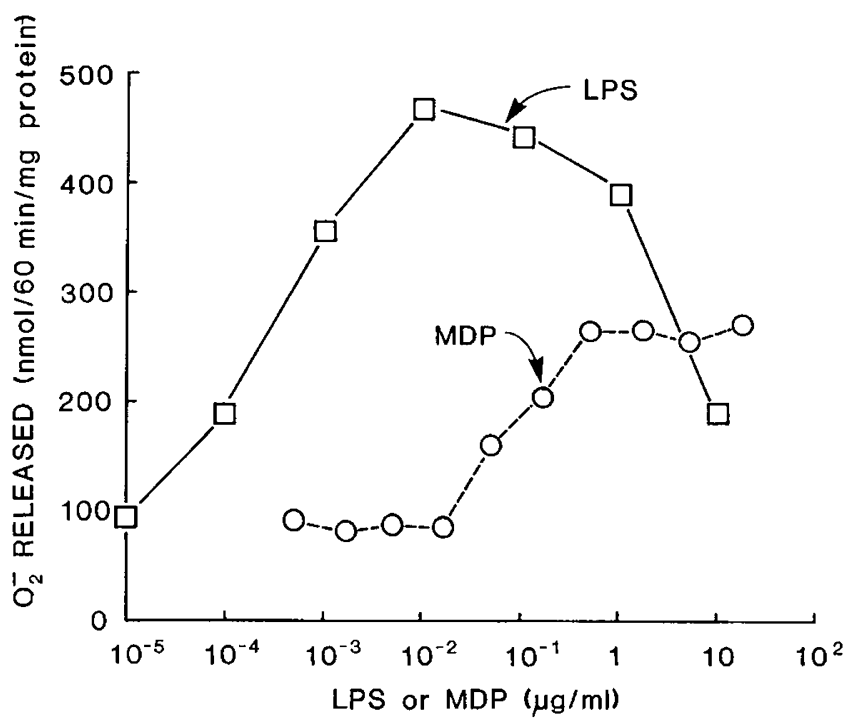

Fig. 3. Effect of concentration of MDP or LPS in the medium on enhancement of the $\mathrm{O}_{2}{ }^{-}$-generating capacity of resident mouse macrophages cultured overnight. Values shown represent the mean $\mathrm{O}_{2}^{-}$produced in response to stimulation with PMA after priming with LPS or $\operatorname{MDP}(n=6, \mathrm{SEM}<15)$. The $\mathrm{O}_{2}$ response of untreated control cultures was $84 \pm 7(n=6)$. (From ref. 9, with permission)

Table 1. Factors capable of priming macrophages for an enhanced respiratory burst

I. With possible therapeutic potential:

Interferon- $\gamma$

Colony-stimulating factor-granulocyte, monocyte

Somatotropin

Muramyl peptides

Vitamin $\mathrm{D}_{3}$

II. Possibly active at local sites:

Tumor necrosis factor $-\alpha$

Platelet-activating factor

Serotonin

Proteases 
of inflammation, as judged primarily by studies in vitro $(7,13-$ 15).

Macrophages are not the only phagocytic cells whose activity can be up-regulated. Neutrophils can also be primed. This capacity to be primed has demonstrated that neutrophils are not an end-stage cell in the functional sense, as had been thought previously (16). Agents that can prime neutrophils for an enhanced respiratory burst include bacterial products (LPS, MDP), chemotactic factors, platelet-activating factor, tumor necrosis factor- $\alpha$, CSF-GM, and interferon- $\gamma(17)$.

Bacterial infection itself can prime neutrophils (18). The priming molecule is not yet understood but might be a product of the infecting organism or any of the other byproducts of inflammation just mentioned. In fact, multiple sources are likely, perhaps depending upon the type of infection. LPS, which is a particularly potent priming agent for neutrophils, also stimulates the release of platelet activating factor and tumor necrosis factor- $\alpha$.

The nitroblue tetrazolium (NBT) test, popular a few years ago as a means of distinguishing bacterial infection from viral infection (19), is a direct manifestation of priming. In that test, the increased reduction of NBT to form blue formazan is a result of the increased production of $\mathrm{O}_{2}^{-}$by primed neutrophils. Unfortunately, the relationship between a positive NBT test and bacterial infection is not consistent enough to aid substantially in the resolution of the common problem of distinguishing between these two classes of infection.

If the molecular basis for the priming phenomenon could be understood, then we might manipulate it intelligently for the purposes of therapy. Any of several possible biochemical changes might underlie the priming or activation of phagocytes (20). It is possible that priming causes an increase in the number or affinity of receptors for the stimuli. This has been studied with several stimuli, but there is no evidence to date that a change in number or affinity of receptors can explain priming. Theoretically, there could be an increase in the content or efficiency of the NADPH oxidase. This appears not to be the case in the primed but unstimulated cell. There is possibly a down-regulator of the NADPH oxidase present on the plasma membrane of monocytes and macrophages, as suggested by the observation that brief protease treatment of these cells can prime them (15).

A fourth possibility, that there might be modification of stimulus-response coupling, or signal transduction, does seem likely. There are reasonably good data to indicate that transduction of the signal induced by stimulus-receptor interaction is faster in a primed cell $(16,17,20)$. This transduction process appears to be greased or "charged" so that it works more efficiently.

There are many steps in the transduction process. None is fully understood. The process might be conceptualized currently as a mosaic. There are pieces of the mosaic that are appreciated at least partially, but understanding of the interactions between the pieces is vague if it exists at all. Currently the fundamental science of cell biology focuses most of its efforts at understanding this process across a variety of cell types. As examples, I will show you experiments relating to only two of these steps.

Figure 4 analyzes the change in electrical potential across the plasma membrane of human neutrophils after stimulation by chemotactic peptide. Membrane potential in this system is measured indirectly by a change in fluorescence of a fluorescent dye, oxonol 5, that partitions into the plasma membrane and does not accumulate in the interior of the cell (17). After stimulation at time zero, normal cells had a very weak hyperpolarization and a very slow depolarization. Cells primed by prior exposure to LPS responded with a vigorous hyperpolarization followed by a pronounced depolarization. The precise basis for this difference is not understood, but the result indicates a much greater capacity of the primed cell for ion flux across the plasma membrane. The consequences to the cell of this difference are likely to be profound.

Figure 5 illustrates that free, unbound $\mathrm{Ca}^{2+}$ inside neutrophils, shown on the vertical axis as fluorescence of the $\mathrm{Ca}^{2+}$-binding

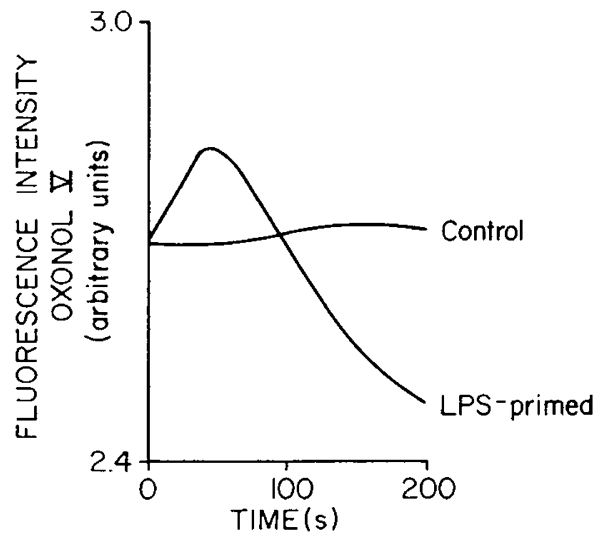

Fig. 4. Time course of change in membrane potential in control and LPS-primed human neutrophils, measured as change of fluorescence intensity of oxonol $\mathrm{V}$. The graph shows superimposed tracings for control and LPS-primed cells stimulated at time 0 by exposure to chemotactic peptide (f-met-leu-phe, $1 \mu \mathrm{M}$ ). (From ref. 17, with permission)
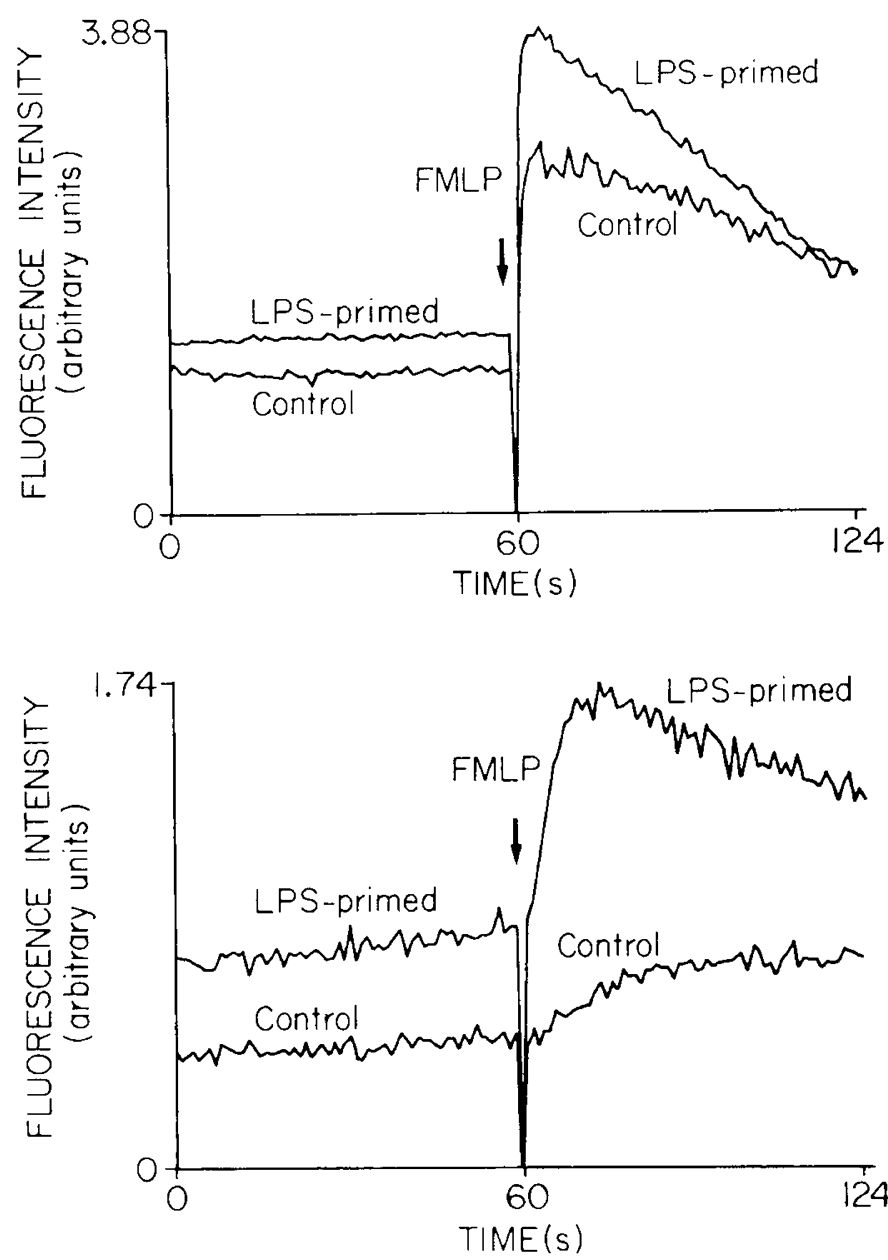

Fig. 5. The effect of LPS priming of human neutrophils on baseline (unstimulated) and poststimulation concentrations of intracellular free $\mathrm{Ca}^{2+}$, measured as fluorescence of fura-2. The chemotactic peptide $\mathrm{f}-$ met-leu-phe (FMLP) was added, as shown by the arrow, at concentrations of $1 \mu \mathrm{M}$ (upper panel) and $1 \mathrm{nM}$ (lower panel). (From Ref. 17, with permission)

dye, fura-2, was higher in unstimulated cells that had been primed with LPS than in control cells. This represents Fura-2 that penetrates the cell and fluoresces in proportion to the amount of $\mathrm{Ca}^{2+}$ bound. Stimulation with chemotactic peptide induced a greater increase in this free $\mathrm{Ca}^{2+}$ in the primed cells. 
Table 2. Treatment of mice infected with $M$. intracellulare with agents that promote macrophage activation*

\begin{tabular}{lc}
\hline \multicolumn{1}{c}{ Treatment } & $\begin{array}{c}\text { CFU/spleen } \\
\text { (millions) }\end{array}$ \\
\hline None & $14.1 \pm 1.7$ \\
Saline & $13.3 \pm 1.3$ \\
Indomethacin $(600 \mu \mathrm{g})$ & $2.0 \pm 0.4$ \\
Muramyl dipeptide $(50 \mu \mathrm{g})$ & $0.4 \pm 0.01$ \\
Interferon- $\gamma(500 \mathrm{U})$ & $0.2 \pm 0.02$ \\
Interferon- $\gamma(2500 \mathrm{U})$ & $0.3 \pm 0.01$ \\
\hline
\end{tabular}

* Mice were given bacteria i.v.; 4 wk later, $8-15$ mice were given the indicated agent i.v. daily for $7 \mathrm{~d}$. Colony-forming units (CFU) are expressed as mean $\pm \mathrm{SEM}, n=8-15$. (Data from ref. 22).

The two panels represent results with two different concentrations of the stimulus. In every experiment, LPS-primed cells had an enhanced respiratory burst (mean of 11 -fold).

Priming for an enhanced respiratory burst can be induced in both neutrophils and macrophages by the relatively $\mathrm{Ca}^{2+}$-specific ionophore ionomycin (21), and priming by LPS can be blocked by preloading the cells with agents that chelate the intracellular free $\mathrm{Ca}^{2+}(17)$. These combined results raise the possibility that the respiratory burst in phagocytic cells is controlled by regulation of intracellular $\mathrm{Ca}^{2+}$, which, in turn, could achieve its effect through modulation of a $\mathrm{Ca}^{2+}$-dependent enzyme or enzymes.

Two recent examples of the manipulation of phagocytes in vivo will illustrate the potential for future development. The first involves a model of chronic myobacterial infection in mice (22). In this model, infection is induced by injection of Mycobacterium avium-intracellulare into the tail vein. There is an initial containment of bacterial replication, followed by accelerated bacterial growth and death of the mice after several months. The primed state of the macrophage, as an index of macrophage activation, increased for about $2 \mathrm{wk}$, as demonstrated by an increase in the release of $\mathrm{O}_{2}^{-}$over that 2 -wk period. This was followed by a surprising decline in the primed state, which was especially pronounced if the macrophages were stimulated by the infecting organism.

Splenic and peritoneal macrophages from infected animals, studied at 2 or $3 \mathrm{wk}$ after the onset of infection, released large amounts of prostaglandin $\mathrm{E}_{2}\left(\mathrm{PGE}_{2}\right)$ when stimulated by antigens from the infecting organisms. $\mathrm{PGE}_{2}$ is known to suppress lymphocyte function. In other experiments, it was found that $\mathrm{PGE}_{2^{-}}$ containing supernates from macrophages of infected mice suppressed the release of interferon from lymphocytes. Thus, it appeared that the microorganism induced macrophage $\mathrm{PGE}_{2}$ release, which inhibited release of macrophage-activating lymphokines (22).

Table 2 indicates that this block in lymphokine release could be circumvented or suppressed. In the experiment shown, after $4 \mathrm{wk}$ of infection, 8-15 mice were injected i.v. daily for $1 \mathrm{wk}$ with one of the agents shown in the left column. Only a graduate student would do this project.

Mice given nothing or saline had 13 to 14 million bacteria per spleen on the eighth day. Injection of indomethacin, which inhibits the synthesis of $\mathrm{PGE}_{2}$, permitted a significant reduction in the infection. The block in release of macrophage-activating lymphokine(s) was circumvented effectively by injection of the macrophage activators, MDP or interferon $-\gamma$. This suppression of the activation process during infection by intracellular parasites may be widespread in nature (22) and may play a role in the chronicity of intracellular infections.

Interferon- $\gamma$, the best defined of the macrophage-activating factors, is beginning to be tested experimentally in humans and has been tried in AIDS, lepromatous leprosy, chronic hepatitis $\mathrm{B}$, condyloma acuminata, chronic myelogenous leukemia, renal cell carcinoma, and under particular circumstances, in CGD (23, 24).

My last example involves the use of interferon- $\gamma$ in CGD.
Interferon- $\gamma$ has had a surprisingly potent effect in patients with this disorder. Esekowitz et al. have shown that interferon- $\gamma$, added in vitro to human phagocytes from patients with cytochrome b-deficient x-linked CGD, induced synthesis of messenger RNA for the major chain of cytochrome $b$ and modest activity of the respiratory burst (25). Thus, in the type of CGD in which the defect lies in an inability to produce normal amounts of cytochrome $b$, interferon- $\gamma$ exhibited a partial capacity to overcome or circumvent the genetic defect in vitro.

On the basis of this result, they injected interferon- $\gamma$ at a dose of $0.1 \mathrm{mg} / \mathrm{m}^{2}$ s.c. on two successive days into two patients who had the classic, cytochrome b-negative, most severe form of $\mathrm{x}$ linked CGD, and two patients with a milder variant of this $\mathrm{x}$ linked form (26). By the eighth day after injection, release of $\mathrm{O}_{2}{ }^{-}$ by stimulated neutrophils was within the normal range in the case of the two patients with the milder disease, and values remained at this level for $30 \mathrm{~d}$. Release of $\mathrm{O}_{2}^{-}$by neutrophils from the two patients with severe disease was affected less by injection of interferon- $\gamma$, but levels still reached $20-30 \%$ of normal at peak on d 15. The patient's phagocytes also demonstrated an increase in messenger RNA transcripts for the heavy chain of cytochrome $b$ and an increased capacity to kill ingested staphylococci. Similar findings to these have been obtained by Gallin et al. at the National Institutes of Health (27), and a large clinical efficacy trial of interferon- $\gamma$ in CGD is now being planned.

Three conclusions appear reasonable. First, pharmacologic upregulation of phagocyte function will permit enhanced rejection of infection and, perhaps, cancer. The capacity to achieve this effect in patients is at hand. Second, it may also be possible in the future to down-regulate phagocytic function under circumstances in which inflammation is detrimental. What seems most certain is that future success in these endeavors will depend upon our gaining further understanding of molecular mechanisms.

\section{REFERENCES}

1. Cerf C, Navasky VS 1984 The Experts Speak. Pantheon Books, New York

2. Curnutte JT (ed) 1988 Phagocytic defects. II. Abnormalities of the respiratory burst. Hematol Oncol Clin North Am 2:185-334

3. Anderson DC, Miller LJ, Schmalstieg FC, Rothlein R, Springer TA 1986 Contributions of the Mac-1 glycoprotein family to adherence-dependent granulocyte functions: structure-function assessments employing subunitspecific monoclonal antibodies. J Immunol 137:15-27

4. Babior BM 1988 The respiratory burst oxidase. Hematol Oncol Clin North Am 2:201-212

5. Segal AW 1988 Cytochrome b-245 and its involvement in the molecular pathology of chronic granulomatous disease. Hematol Oncol Clin North Am 2:213-223

6. Dinauer MC, Orkin SH, Brown R, Jesaitis AJ, Parkos CA 1987 The glycoprotein encoded by the $\mathrm{x}$-linked chronic granulomatous disease locus is a component of the neutrophil cytochrome b complex. Nature 327:717-720

7. Johnston Jr RB 1988 Current concepts: immunology-monocytes and macrophages. N Engl J Med 318:747-752

8. Johnston Jr RB, Godzik CA, Cohn ZA 1978 Increased superoxide anion production by immunologically activated and chemically elicited macrophages. J Exp Med 148:115-127

9. Pabst MJ, Johnston Jr RB 1980 Increased production of superoxide anion by macrophages exposed in vitro to muramyl dipeptide or lipopolysaccharide. J Exp Med 151:101-114

10. Reed SG, Nathan CF, Pihl DL, Rodricks P, Shanebeck K, Conlon PJ, Grabstein KH 1987 Recombinant granulocyte/macrophage colony-stimulating factor activates macrophages to inhibit Trypanosoma cruzi and release hydrogen peroxide: comparison with interferon- $\gamma$. J Exp Med 166:1734-1746

11. Cummings NP, Pabst MJ, Johnston Jr RB 1980 Activation of macrophages for enhanced release of superoxide anion and greater killing of Candida albicans by injection of muramyl dipeptide. J Exp Med 152:1659-1669

12. Cohen MS, Mesler DE, Snipes RG, Gray TK 1986 1,25-Dihydroxyvitamin D activates secretion of hydrogen peroxide by human monocytes. $\mathbf{J}$ Immunol 136:1049-1053

13. Edwards CK III, Ghiasuddin SM, Schepper JM, Yunger LM, Kelley KW 1988 A newly defined property of somatotropin: priming of macrophages for production of superoxide anion. Science 239:769-771.

14. de Titto EH, Catterall JR, Remington JS 1986 Activity of recombinant tumor necrosis factor on Toxoplasma gondii and Trypanosoma cruzi. $\mathrm{J}$ Immunol 137:1342-1345

15. Speer CP, Pabst MJ, Hedegaard HB, Rest RF, Johnston Jr RB 1984 Enhanced release of oxygen metabolites by monocyte-derived macrophages exposed to 
proteolytic enzymes: activity of neutrophil elastase and cathepsin G. J Immunol 133:2151-2156

16. Guthrie LA, McPhail LC, Henson PM, Johnston Jr RB 1984 Priming of neutrophils for enhanced release of oxygen metabolites by bacterial lipopolysaccharide: evidence for increased activity of the superoxide-producing enzyme. J Exp Med 160:1656-1671

17. Forehand JR, Pabst MJ, Phillips WA, Johnston Jr RB 1989 Lipopolysaccharide priming of human neutrophils for an enhanced respiratory burst: role of intracellular free calcium. J Clin Invest 83:74-83

18. Bass DA, Olbrantz P, Szejda P, Seeds MC, McCall CE 1986 Subpopulations of neutrophils with increased oxidative product formation in blood of patients with infection. J Immunol 136:860-866

19. Park BH, Fikrig SM, Smithwick EM 1968 Infection and nitroblue-tetrazolium reduction by neutrophils: a diagnostic aid. Lancet 2:532-534

20. Johnston Jr RB, Kitagawa S 1985 Molecular basis for the enhanced respiratory burst of activated macrophages. Fed Proc 44:2927-2932

21. Finkel TH, Pabst MJ, Suzuki H, Guthrie LA, Forehand JR, Phillips WA, Johnston Jr RB 1987 Priming of neutrophils and macrophages for enhanced release of superoxide anion by the calcium ionophone ionomycin: implications for regulation of the respiratory burst. J Biol Chem 262:12589-12596

22. Edwards CK III, Hedegaard HB, Zlotnik A, Gangadharam PR, Johnston Jr
RB, Pabst MJ 1986 Chronic infection due to Mycobacterium intracellulare in mice: association with macrophage release of prostaglandin $E_{2}$ and reversal by injection of indomethacin, muramyl dipeptide, or interferon- $\gamma$. J Immunol 136:1820-1827

23. Nathan CF, Horowitz CR, de la Harpe J, Vadhan-Raj S, Sherwin SA, Oettingen $\mathrm{HF}$, Krown SE 1985 Administration of recombinant interferon- $\gamma$ to cancer patients enhances monocyte secretion of hydrogen peroxide. Proc Natl Acad Sci USA 82:8686-8690

24. Jaffe HS, Sherwin SA 1986 The early clinical trials of recombinant human interferon-gamma. In: Friedman RM (ed) Interferons as Cell Growth Inhibitors and Antitumor Factors. Alan R Liss, New York, pp 509-522

25. Esekowitz RAB, Orkin SH, Newburger PE 1987 Recombinant interferon gamma augments phagocyte superoxide production and $\mathrm{x}$-chronic granulomatous disease gene expression in $x$-linked variant chronic granulomatous disease. J Clin Invest 80:1009-1016

26. Esekowitz RAB, Dinauer MC, Jaffe HS, Orkin SH, Newburger PE 1988 Partia correction of the phagocyte defect in patients with $\mathrm{x}$-linked chronic granulomatous disease by subcutaneous interferon gamma. $N$ Engl $\mathrm{J}$ Med 319:146-151

27. Gallin JI, Sechler JMG, Malech HL 1988 Recombinant human interferon- $\gamma$ reconstitutes defective phagocyte function in patients with chronic granulomatous disease of childhood. Clin Res 36:620A(abstr) 